\title{
AHP difuso y TOPSIS para la selección de un proveedor 3PL considerando el riesgo operacional
}

\author{
NATALia TABAREs-URreA ${ }^{1}$ \\ GISELLE RAMÍREZ-FLóREZ ${ }^{2}$ \\ JUAN C. OSORIO-GÓMEZ
}

\section{Resumen}

La contratación de proveedores de tercera parte logística (3PL) ha venido aumentando en los últimos años a nivel mundial y local. Uno de los servicios que más se terceriza es el transporte. La contratación de un proveedor de tercera parte para el transporte terrestre de mercancías es un problema de decisión multicriterio que debe ser abordado con herramientas adecuadas de manera que se garantice la mejor contratación posible considerando los criterios que la organización contratante considere para esta transacción. El presente trabajo propone un desarrollo multicriterio a partir de la utilización del proceso analítico jerárquico difuso (AHP difuso) y TOPSIS para la selección de un proveedor de tercera parte en el transporte terrestre de mercancía incluyendo el riesgo operacional como uno de los criterios de selección. La propuesta es aplicada para la selección de un transportador por parte de una empresa manufacturera del Valle del Cauca en Colombia.

Palabras Clave: Selección de proveedores, Tercera parte logística - 3PL, AHP difuso, TOPSIS, Transporte terrestre de mercancías, Problemas de decisión multicriterio, Riesgo operacional.

\section{Diffuse AHP and TOPSIS for the selection of a third-party provider considering operational risk}

\section{Abstract}

Contracting third-party logistics providers (3PL) is an activity that has been increasing over the last years globally and locally. One of the most outsourced services is transportation. Hiring a 3PL for ground transportation of merchandise

\footnotetext{
Geiico SA.

Omnilife Manufactura de Colombia SAS.

Escuela de Ingeniería Industrial, Universidad del Valle. Cali, Colombia.
}

Autor de correspondencia: Osorio Gómez, J.C. (Juan Carlos): Universidad del Valle, Escuela de Ingeniería Industrial: Cra 13 \# 100 - 00, 760001. Teléfono: 3176563427. Correo electrónico: Juan.osorio@correounivalle.edu.co
Historia del artículo:

Artículo recibido: 07-VI-2019/ Aprobado: 15-I-2020

Disponible online: 15 de enero de 2020

Discusión abierta hasta septiembre de 2021 
is a multi-criteria decision-based problem, which must be addressed with the right tools in order to guarantee the best possible hiring taking into account the criteria that the contracting organization considers the most important for this transaction. The present paper proposes a multi-criteria development based on the use of the diffuse hierarchical analytical process (diffuse AHP) and TOPSIS for the selection of a third-party supplier in ground transportation of merchandise, including operational risk as one of the selection criteria. The proposal is applied to the selection of a transporter by a manufacturing company in Valle del Cauca, Colombia.

Keywords: Selection of suppliers, Third-party logistics 3PL, Diffuse AHP, TOPSIS, Ground transportation of merchandise, Multicriteria decision problems, Operational risk.

\section{AHP difuso e TOPSIS para a seleção de um provedor 3PL considerando o risco operacional}

\section{Resumo}

A contratação de provedores logísticos de terceiros (3PL) vem aumentando nos últimos anos globalmente e localmente. Um dos serviços mais terceirizados é o transporte. A contratação de um terceiro fornecedor para o transporte terrestre de mercadorias é um problema de decisão multicritério que deve ser tratado com ferramentas adequadas para garantir a melhor contratação possível considerando os critérios que a organização contratante considera para essa transação. 0 presente trabalho propõe um desenvolvimento multicritério baseado na utilização do processo analítico hierárquico difuso (AHP difuso) e TOPSIS para a seleção de um terceiro fornecedor no transporte terrestre de mercadorias, incluindo o risco operacional como um dos critérios de seleção. A proposta é aplicada para a seleção de um transportador por uma empresa de fabricação em Valle del Cauca na Colômbia.

Palavras chave: Seleção de fornecedores, Terceira parte logística - 3PL, AHP difuso, TOPSIS, Transporte terrestre de marcadorias, Problemas de decisão multicitério, Risco operacional.

\section{Introducción y revisión de la literatura}

La contratación de proveedores 3PL ha sido impulsada en gran medida por el fenómeno de crecimiento del outsourcing logístico, que se atribuye a los beneficios que brinda en términos de reducción de costos y mejoramiento del desempeño, enfocándose en sus actividades principales y en la construcción de alianzas estratégicas apoyando la cadena de suministro (Aguezzoul 2014; Lee et al. 2012). Por ello, la selección y evaluación de proveedores 3PL se ha convertido en una actividad importante en el proceso de subcontratación (Ho et al. 2012; Karthik. et al. 2015; Alkhatib et al. 2015).

Aguezzoul (2014) muestra que la selección de un proveedor 3PL es una decisión multicriterio porque requiere tomar en cuenta varios criterios. Algunos de ellos son desarrollados para las necesidades específicas de los clientes, mientras que otros son comunes para todas las circunstancias. Sin embargo, según lo argumentado por Ho et al. (2011), generalmente no se considera en la identificación de criterios, los impactos en los objetivos y requisitos de las partes interesadas de la empresa, lo cual hace que el proveedor seleccionado pueda no cumplir los requerimientos de la compañía.

Generalmente, cada actividad de negocio implica riesgos. Debido a que la contratación de proveedores logísticos es una actividad de outsourcing, las compañías pueden verse afectadas por los riesgos que impactan en la cadena de suministro y que han tomado una gran importancia en estudios realizados desde el 2008 al 2013 (Saleh Fahed Alkhatib, 2015). Varios autores especifican los riesgos en que puede incurrir la compañía al contratar un proveedor 3PL, que dependerán de las actividades para las que será contratado dicho proveedor. La gestión del riesgo en la cadena de suministro ha surgido como un área importante de investigación en la gestión de la 
misma (Aqlan and Lam, 2015). Tang (2006) define la gestión de los riesgos en la cadena de suministro (SCRM por sus siglas en inglés) como la gestión de los riesgos a través de coordinación y colaboración a lo largo de la cadena con los socios, para asegurar la rentabilidad y la continuidad de la misma. Götze and Mikus, 2007; Jüttner et al. 2003 (citados por Pfohl et al.2011), proponen que los riesgos pueden ser divididos en tres áreas que se relacionan de acuerdo con su punto de origen: riesgos dentro de la empresa, riesgos fuera de la empresa pero dentro de la cadena y riesgos fuera de la cadena. Este trabajo de investigación se enfoca en los riesgos que están vinculados tanto en la empresa como en los eslabones asociados a la cadena.

La contratación de proveedores 3PL aporta una gran diferenciación en la cadena de suministro de una empresa, pues esta adquiere servicios enfocados hacia una necesidad específica sin tener que invertir en ellos, además de que le ayuda a ganar valor para sus clientes (Arroyo, Gaytan and Sierra, 2007).

Seleccionar proveedores de manera acertada es una tarea compleja a la que se enfrentan día a día las organizaciones, ya que si esta falla en dicha selección, se pueden presentar consecuencias que afectan directamente su rendimiento, su productividad y su competitividad.

A medida que se amplía el rango de servicios que prestan los proveedores 3PL o cuando la actividad logística que se terceriza deja de ser rutinaria y de soporte, se aumenta la dependencia de la empresa hacia el proveedor, lo que incrementa el riesgo asociado a una mala decisión en la selección de la tercera parte (Arroyo, Gaytan and Sierra, 2007).

Además, seleccionar un proveedor es una decisión estratégica y de largo plazo. Una relación de largo plazo con los proveedores resulta beneficiosa para una compañía porque ofrece ventajas como la estabilidad en los planes y estrategias en el futuro, la fiabilidad en la calidad del producto y tiempo del servicio, una mejor comprensión de la cadena de suministro, posibles descuentos y una mejor gestión de inventarios (Osorio, Manotas and García, 2016). La elección de los proveedores no debe depender únicamente de su elegibilidad en el momento de la toma de la decisión; es muy importante analizar cambios pasados y predecir situaciones en el futuro, pues esto disminuye la necesidad de evaluar y reemplazar periódicamente los contratos existentes (Hosseininasab and Ahmadi, 2015).

De acuerdo con Kumar \& Singh (2012) el 55\% de las alianzas logísticas terminaron después de tres a cinco años de ser contratados. En algunos casos se observó que las relaciones terminaban incluso antes de completar el primer año de operación. Las relaciones fracasadas con los proveedores 3PL son el resultado de un pobre proceso de selección, pocas o inexistentes estrategias de subcontratación, coordinación ineficiente durante la integración al proceso y gestión inapropiada en la evaluación de la relación. Antes de elegir un proveedor 3PL se deben definir y evaluar una serie de criterios de acuerdo con los requerimientos.

La decisión de contratar proveedores 3PL depende de una variedad de factores como calidad del servicio, capacidad de respuesta, flexibilidad, fiabilidad, la estabilidad financiera, la reputación del proveedor, referencias de clientes, el apoyo a la alta dirección, el riesgo, economías de escala, entre otros, los cuales difieren según cada organización. La decisión de subcontratar ciertas funciones de la cadena de suministro, dependerá de los planes de la compañía, los objetivos futuros, las líneas de productos, la expansión y las adquisiciones de la organización (Rattanawiboonsom, 2014). Al mismo tiempo, las amenazas en el mercado, tales como los efectos de la globalización, la recesión económica y la sostenibilidad, incrementan los niveles de incertidumbre y motivan a las organizaciones a replantearse la manera de evaluar y seleccionar a sus proveedores (Alkhatib et al. 2015; Kumar \& Singh 2012).

Respecto a la selección de estos proveedores, muchos autores han realizado estudios para elegir los proveedores adecuados usando diferentes técnicas como modelos de programación matemática, métodos multicriterio (MCDM) y algoritmos inteligentes (Perçin, 2009). En la búsqueda realizada para este artículo, se encontró entre otras cosas, que las técnicas multicriterio más utilizadas en el proceso de selección y evaluación del desempeño son la Lógica Difusa (Fuzzy logic), seguida del Proceso Analítico Jerárquico (AHP), la Técnica para el Orden de preferencia por similitud con solución ideal (TOPSIS), y las técnicas híbridas como el AHP difuso (FAHP). 
TABLA 1. CRITERIOS DE SELECCIÓN PARA ACTIVIDADES DE TRANSPORTE TERCERIZADO

FUENTE: ELABORACIÓN PROPIA

\begin{tabular}{|c|c|c|c|c|c|c|c|c|c|c|c|c|c|c|c|c|c|c|c|c|}
\hline & & \multicolumn{19}{|c|}{ Criterios } \\
\hline \multirow{10}{*}{$\begin{array}{l}z \\
\text { 은 } \\
\text { ư } \\
\text { un }\end{array}$} & Autores & 1 & 2 & 3 & 4 & 5 & 6 & 7 & 8 & 9 & 10 & 11 & 12 & 13 & 14 & 15 & 16 & 17 & 18 & 19 \\
\hline & Chan \& Kumar (2005) & $x$ & & $x$ & & $x$ & & & $x$ & $x$ & & & $x$ & & & $x$ & & & $x$ & \\
\hline & Aguezzoul (2010) & $x$ & $x$ & $x$ & $x$ & $x$ & $x$ & $x$ & $x$ & $x$ & & $\mathrm{x}$ & $x$ & & & $x$ & & & & \\
\hline & Cooper et al. (2012) & $\mathrm{x}$ & $x$ & $x$ & & & $x$ & & & & $x$ & & & & & & & & & \\
\hline & Daim et al. (2012) & $x$ & & & & $x$ & $x$ & $x$ & & $x$ & & $x$ & & & $x$ & & $x$ & & & \\
\hline & Bansal \& Kumar (2013) & $x$ & $x$ & $x$ & & $x$ & $x$ & $x$ & & & & & $x$ & $x$ & & $x$ & & & & \\
\hline & Rattanawiboonsom (2014) & & $x$ & $x$ & $x$ & & $x$ & & $x$ & $x$ & $x$ & & $x$ & & & & & & & \\
\hline & Aguezzoul (2014) & $\mathrm{x}$ & $x$ & $x$ & $x$ & $x$ & $x$ & $x$ & $x$ & $x$ & & $x$ & $x$ & & & $x$ & & & & \\
\hline & Alkhatib et al. (2015) & $x$ & $x$ & $x$ & $x$ & & $x$ & $x$ & & & $x$ & & & & & & & & & $x$ \\
\hline & Lieb \& Bentz (2015) & $x$ & $x$ & $x$ & $x$ & $x$ & & & & & & & & & & & & & & \\
\hline
\end{tabular}

\section{Criterios}

1: Costo/Precio, 2: Confiabilidad, 3: Calidad, 4: Flexibilidad, 5: Tecnologías de información, 6: Servicio, 7: Localización geográfica, 8: Estabilidad financiera, 9: Reputación, 10: Riesgo, 11: Experiencia, 12: Comunicación, 13: Sostenibilidad, 14: Equipo Físico, 15: Entregas a tiempo, 16: Aspectos culturales, 17: Capacidad de almacenamiento, 18: Capacidades de resolución de problemas, 19: Innovación en servicios.

\section{Criterios de selección de proveedores logísticos}

En la Tabla 1 se pueden observar los criterios de transporte, definidos por los autores que se han revisado de acuerdo con la búsqueda bibliográfica realizada y entre los que se destacan el costo, la confiabilidad y la calidad.

Uno de los principales problemas a los que se ve enfrentada la alta dirección, se debe a la incorporación de medidas de riesgo en el proceso de selección de proveedores (Kull and Talluri, 2008). Al tratarse de una decisión de carácter estratégico, que supone ser de largo plazo, la hace aún más compleja. Por otra parte, las relaciones de largo plazo, que incluyen riesgos y beneficios compartidos, garantizan la cooperación entre el usuario y el proveedor y, por ende, ayudan a tener un control oportuno de los proveedores.

Varios autores especifican los riesgos en que puede incurrir la compañía al contratar un proveedor 3PL, y otros los categorizan de acuerdo con su forma y medio de presentación. Por ello, tener una buena gestión de los riesgos es un elemento clave para las organizaciones porque les permite manejar y contrarrestar factores de incertidumbre y con ello, lograr el éxito sostenido de la misma en el tiempo.

\section{La tercerización y los riesgos en la cadena de suministro}

Schoenherr et al. (2008) aseguran que la tercerización puede crear una red de suministro más compleja con mayores riesgos, sobre todo en la cadena de suministro. Estos riesgos, que pueden incluir la mala calidad, mayores costos de transporte, menor fiabilidad, interrupciones en el suministro, fallas logísticas, desastres naturales y aumento de las dificultades de comunicación, deben ser reconocidos y analizados frente a las ventajas de realizar sus propias operaciones.

Los factores que contribuyen a la probabilidad de amenazas de riesgo según Aqlan \& Lam (2015) son: (i) la globalización de suministros, la producción y las ventas, (ii) el incremento de la complejidad y la competitividad y (iii) ocurrencia de eventos de riesgo internos y externos tales como la escasez de material y los desastres naturales. 
Kull \& Talluri (2008), Lee et al. (2012) y Chen \& Wu (2013), afirman que el riesgo de suministro está asociado con fallas en las entregas, costos, calidad, capacidad de producción, flexibilidad y fallas de confianza en general, entre otros. Similarmente, Tylor et. al. citados por Wu et al. (2006) dicen que el riesgo es inherente a este tipo de sistemas y puede manifestarse en la incertidumbre existente en los requerimientos de la demanda, capacidad, tiempo de entrega, el tiempo de fabricación y los costos.

Tang \& S. Nurmaya (2011) describen que los riesgos en la cadena de suministro están compuestos por riesgos operacionales y riesgos disruptivos. Los riesgos operacionales están asociados con la incertidumbre de un proceso, tal como demanda de los clientes, la cantidad a suministrar, y las fluctuaciones de los costos. Los riesgos disruptivos abarcan desastres naturales y humanos, tales como terremotos, inundaciones, huracanes, ataques terroristas, epidemias, crisis financieras, huelgas e inestabilidad política.

\section{Riesgos en las actividades logísticas.}

La decisión de seleccionar un proveedor en una cadena de suministro no depende únicamente de medidas referentes al costo o la calidad, también depende de diversos factores de riesgo (Osorio, Manotas and García, 2016). Estos factores están asociados a la planeación, la calidad, la flexibilidad, el historial y la confiabilidad que brindan dentro de sus servicios a diversos clientes.

En la Tabla 2 se muestran los riesgos operacionales asociados a los servicios de tercerización, identificados en la revisión de la literatura.

Respecto a las herramientas utilizadas para la selección de proveedores 3PL, de acuerdo con la revisión realizada sobre las herramientas utilizadas para la selección de proveedores 3PL, se ha construido la Tabla 3. En ella se observa que la lógica difusa y el AHP están entre las más utilizadas. También se observa que es cada vez más frecuente la utilización de combinación de las herramientas.

\section{TABLA 2. PRINCIPALES RIESGOS EN LAS ACTIVIDADES DE TRANSPORTE}

FUENTE: ELABORACIÓN PROPIA

\begin{tabular}{|c|c|}
\hline Riesgos & Autores \\
\hline Pérdida de control & (Perçin, 2009, 2008; Lee, 2009; Huo, Ye and Zhao, 2015) \\
\hline Seguridad e información & $\begin{array}{l}\text { (Perçin, 2008; Manotas Duque, Osorio Gómez and Rivera, 2016; Govindan and } \\
\text { Chaudhuri, 2015; Nekooie, Sheikhalishahi and Hosnavi, 2015; Huo, Ye and Zhao, 2015) }\end{array}$ \\
\hline Económicos & $\begin{array}{l}\text { (Perçin, 2008; Kull and Talluri, 2008; Aqlan and Lam, 2015; Nekooie, Sheikhalishahi } \\
\text { and Hosnavi, 2015; Lee, 2009; Govindan and Chaudhuri, 2015; Chan and Kumar, 2007; } \\
\text { Manotas Duque, Osorio Gómez and Rivera, 2016) }\end{array}$ \\
\hline Comunicación & $\begin{array}{l}\text { (Perçin, 2008; Govindan and Chaudhuri, 2015; Lee, 2009; Manotas Duque, Osorio } \\
\text { Gómez and Rivera, 2016) }\end{array}$ \\
\hline $\begin{array}{c}\text { Complejidad de las } \\
\text { operaciones y entrega }\end{array}$ & (Perçin, 2009; Kull and Talluri, 2008; Manotas Duque, Osorio Gómez and Rivera, 2016) \\
\hline $\begin{array}{l}\text { Flexibilidad en las } \\
\text { operaciones }\end{array}$ & (Jharkharia and Shankar, 2007; Kull and Talluri, 2008; Govindan and Chaudhuri, 2015) \\
\hline Calidad & (Kull and Talluri, 2008; Govindan and Chaudhuri, 2015) \\
\hline $\begin{array}{l}\text { Falta de experiencia y mala } \\
\text { reputación }\end{array}$ & (Govindan and Chaudhuri, 2015; Lee, 2009) \\
\hline Falta de confianza & (Kull and Talluri, 2008; Govindan and Chaudhuri, 2015) \\
\hline Manipulación excesiva & (Nekooie, Sheikhalishahi and Hosnavi, 2015) \\
\hline Entregas fuera de tiempo & (Jharkharia and Shankar, 2007; Govindan and Chaudhuri, 2015) \\
\hline Planeación & (Govindan and Chaudhuri, 2015) \\
\hline $\begin{array}{l}\text { Falta de cobertura y } \\
\text { ubicación geográfica }\end{array}$ & (Govindan and Chaudhuri, 2015; Chan and Kumar, 2007) \\
\hline
\end{tabular}




\begin{tabular}{|c|c|}
\hline HERRAMIENTAS & AUTORES REVISADOS \\
\hline LÓGICA DIFUSA & $\begin{array}{l}\text { (Sahu et al. 2015) (Wang \& Lv 2015)(Saleh Fahed Alkhatib, 2015)(Hwang and Shen, } \\
\text { 2015)(Aguezzoul, 2014)( A. Aguezzoul, 2010)( Bansal, Kumar, \& Issar 2013)(Hwang and } \\
\text { Shen, 2015)(Li et al., 2012)(Liu and Wang, 2009)(Hassanzadeh and Razmi, 2009)(Ne- } \\
\text { kooie, Sheikhalishahi and Hosnavi, 2015) }\end{array}$ \\
\hline AHP & $\begin{array}{l}\text { (Hwang, Chen and Lin, 2016)(Saleh Fahed Alkhatib, 2015)(Daim, Udbye and Balasu- } \\
\text { bramanian, 2013)(Bansal and Kumar, 2013)(Aguezzoul, 2014)(Bayazit \& karpak 2013) } \\
\text { (Shiang et al., 2011)( A. Aguezzoul 2010)(Senthil, Srirangacharyulu and Ramesh, 2012) } \\
\text { (Kull and Talluri, 2008) }\end{array}$ \\
\hline AHP DIFUSO (FAHP) & $\begin{array}{l}\text { (Chan and Kumar, 2007)(Lee, 2009)(Kilincci and Onal, 2011)(Chang, 1996)(Ayhan, 2013) } \\
\text { (Demirel and Kahraman, 2008)(Dadeviren and Yuksel, 2008)(Chang, Wu and Chen, 2008) } \\
\text { (Zapata, 2011)(Govindan and Murugesan, 2011)(Datta et al., 2013)(Soh, 2010) }\end{array}$ \\
\hline FAHP Y TOPSIS & $\begin{array}{l}\text { (Kumar and Singh, 2012)(Akman and Baynal, 2014)(Osorio, Manotas and García, 2016) } \\
\text { (Urbano Guerrero, Muñoz Marín and Osorio Gómez, 2015)(Perçin, 2008) }\end{array}$ \\
\hline TOPSIS & $\begin{array}{l}\text { (Saleh Fahed Alkhatib, 2015)(Aguezzoul, 2014)( A. Aguezzoul 2010)(Senthil, Sriran- } \\
\text { gacharyulu and Ramesh, 2012)customer expect manufacturer to develop a reverse } \\
\text { logistics system so that the retur }\end{array}$ \\
\hline ANP & $\begin{array}{l}\text { (Aguezzoul 2011) (Aguezzoul (2014) (Alkhatib et al., 2014) (Jharkharia \& Shankar 2007) } \\
\text { (Nekooie et al. 2015)(Cooper, Tadikamalla and Shang, 2012) }\end{array}$ \\
\hline $\begin{array}{l}\text { DEMATEL (Decision- making } \\
\text { trial and evaluation laboratory) }\end{array}$ & $\begin{array}{l}\text { (Aguezzoul 2011) (Aguezzoul 2014) (Alkhatib et al. 2014) (Uygun, Kacamak \& Kahraman } \\
\text { 2014) }\end{array}$ \\
\hline AHPYTOPSIS & $\begin{array}{l}\text { (Perçin 2009) (Jayant, Gupta, Garg, \& Khan 2014) (Senthil, Srirangacharyulu and Ra- } \\
\text { mesh, 2012) }\end{array}$ \\
\hline DEA & (Aguezzoul 2011) (Alkhatib et al. 2014) (Amin \& Razmi 2009) \\
\hline FANP & (Chunhao, Yonghe, \& Yuanwei 2008) (Uygun et al. 2014) (Soh 2010) \\
\hline $\begin{array}{l}\text { VIKOR (Multicriteria optimiza- } \\
\text { tion and compromise solution) }\end{array}$ & (Aguezzoul 2011) (Aguezzoul 2014) \\
\hline $\begin{array}{l}\text { ELECTRE (Elimination and } \\
\text { Choice Expressing Reality) }\end{array}$ & (Aguezzoul 2011) (Aguezzoul 2014) \\
\hline TEORÍA DE UTILIDAD & (Aguezzoul 2011) (Aguezzoul 2014) \\
\hline $\begin{array}{l}\text { QFD (Quality Function Devel- } \\
\text { opment) }\end{array}$ & (Aguezzoul 2014)(Karsak \& Dursun, 2014) \\
\hline
\end{tabular}

\section{Propuesta metodológica para la} selección de proveedores 3PL considerando el riesgo

En la Figura 1 se presenta la propuesta metodológica desarrollada para lograr la selección de un proveedor 3PL en la que se considere el riesgo operacional dentro de los criterios de selección.

El esquema metodológico para la selección de proveedores 3PL, está conformado por dos etapas. La primera es la aplicación del AHP difuso con el fin de determinar el peso de los criterios que se evaluarán para la toma de 
decisiones. Esta es una actividad de marco estratégico, pues se realiza cada vez que la organización decida revisar los criterios y los pesos asociados a estos. La segunda, es la aplicación de la herramienta TOPSIS, con el fin de evaluar las alternativas, y decidir cuál de ellas es la que mejor se ajusta a los requerimientos de la compañía. Esta es una actividad táctica y se realiza cada vez que se tenga que decidir sobre la contratación del proveedor 3PL.

\section{Figura 1. Esquema metodológico para la selección de proveedores $3 \mathrm{PL}$}

\section{Fuente: Elaboración propia}

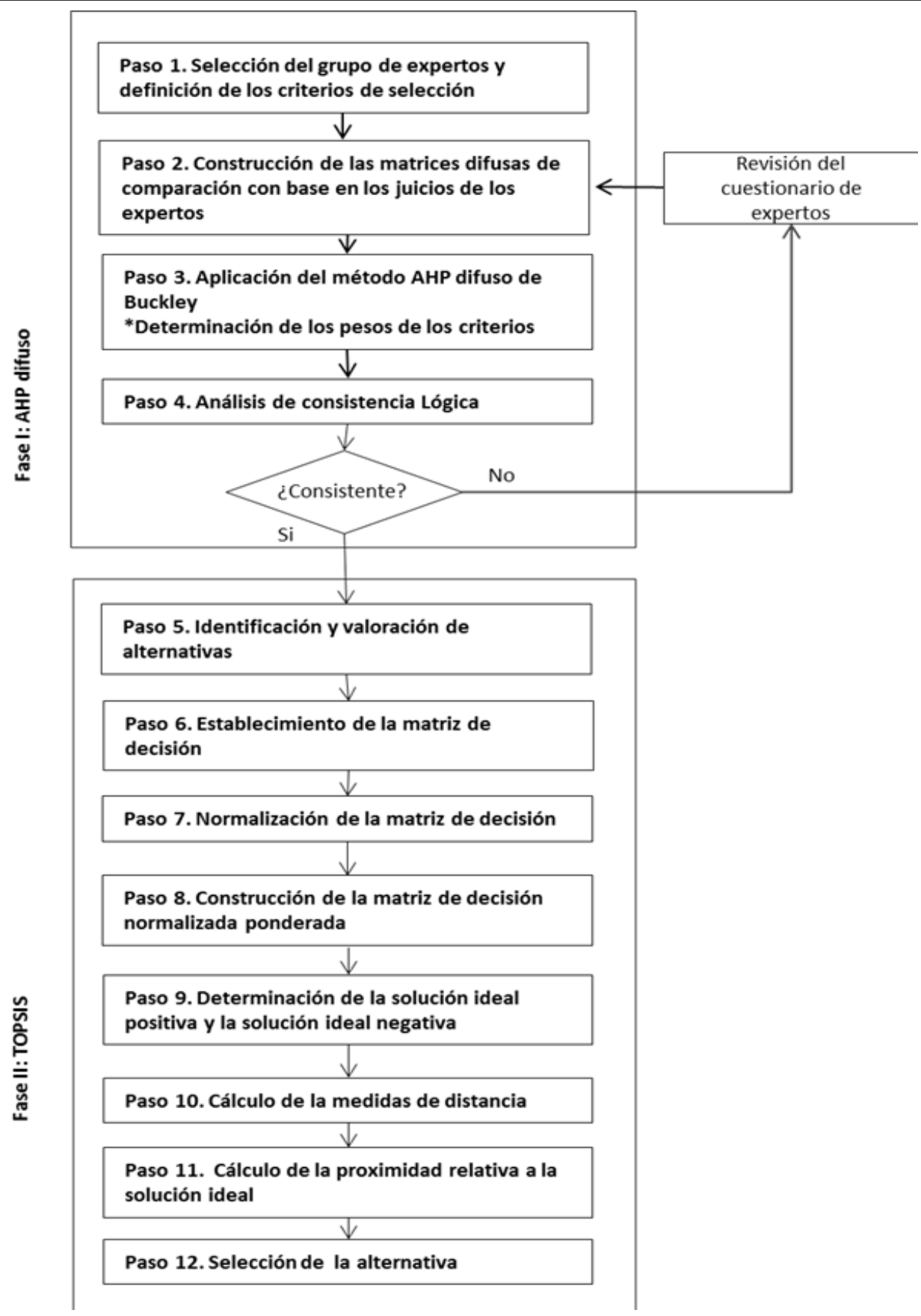




\section{Fase I. Metodología AHP difuso}

\section{Paso 1. Selección del grupo de expertos y} definición de los criterios

La selección del grupo de expertos es un proceso cuidadoso, en el que se deben escoger a las personas que están directamente involucradas a él, puesto que son ellas quienes conocen las necesidades y la operación correctamente, y pueden verse más afectadas por dichas inversiones o decisiones. Además, la razón básica del por qué el grupo de expertos es esencial para el método AHP difuso, se debe a que son ellos los encargados tanto de definir la estructura jerárquica del problema, como de realizar las comparaciones entre los criterios de la misma (Zapata, 2011).

La metodología AHP tradicional se fundamenta a través de la estructuración de un modelo jerárquico: relación existente entre el objetivo o metas, y los criterios y alternativas en el proceso de decisión. Para esta propuesta dicha estructura jerárquica tiene alcance hasta la definición del peso de los criterios, puesto que para la evaluación de las alternativas se utiliza la herramienta TOPSIS.

La selección de los criterios se hace a través de los juicios emitidos por los expertos, los cuales son conocidos mediante la aplicación de un cuestionario, en el cual se consideran los criterios comúnmente utilizados según la revisión bibliográfica mostrada anteriormente.
Una vez las valoraciones de los criterios son obtenidas y promediadas, estas se organizan en orden descendente, tomando aquellas que tienen los resultados más altos.

En la Figura 2 se muestra la representación jerárquica propia de esta metodología: el nivel 1 corresponde al objetivo o meta, el nivel 2 a los criterios y el nivel 3 a las alternativas (evaluadas a través de la aplicación de TOPSIS).

\section{Paso 2. Construcción de las matríces} difusas de comparación con base en los juicios de los expertos

La matriz de comparación se construye con el fin de obtener los juicios de los expertos sobre los criterios definidos en el paso anterior.

Paso 3. Aplicación del método AHP difuso (metodología de Buckley)

Consolidada la matriz difusa de decisión, se halla el vector de prioridad " $\mathrm{N}_{\mathrm{i}}$ ", el cual define la ponderación de cada uno de los criterios definidos en el paso 1 . Posteriormente, se halla el inverso de la suma (verticalmente). Este valor, se multiplica por cada uno de los valores del vector " $r_{i}$ " para obtener el vector ponderado " $w_{i}$ ". Finalmente, el vector resultante " $\mathrm{M}_{\mathrm{i}}$ " se lleva a números nítidos mediante la ecuación propuesta en el método de Buckley (1985).

\section{Figura 2. Representación jerárquica de la metodología propuesta para la selección de proveedores 3PL.}

\section{Fuente: Elaboración propia}

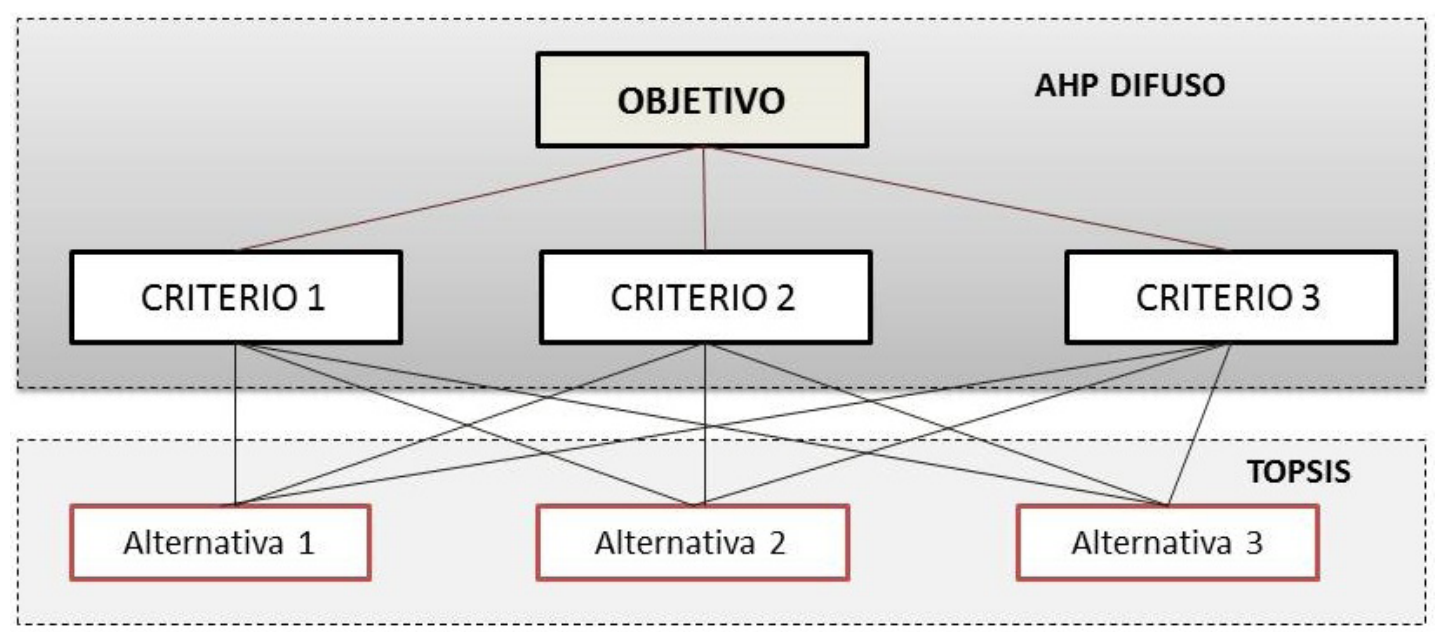




\section{Paso 4. Análisisis de consistencia lógíca}

El método AHP normalmente utiliza un índice de consistencia, que mide la consistencia de los juicios de cada matriz de comparación para toda la jerarquía. Según Saaty (Saaty, 1987), este valor no puede ser mayor a 0,1 (para que sea consistente), con lo que se asegura la consistencia de los juicios del panel de expertos que los realiza.

El proceso de análisis de consistencia inicia con la defuzzificación de la matriz de comparación, es decir, llevar los elementos a números nítidos. Una vez realizado este paso, el proceso es el mismo que para calcular el índice de consistencia del AHP tradicional.

\section{Fase II. Metodología TOPSIS}

\section{Paso 5. Identificación y valoración de las allernativas}

Aquí, se identifica el conjunto finito de alternativas con base en la estructura jerárquica y se procede a determinar los aspectos que se evaluarán según los criterios establecidos en la fase I.

Para poder llevar a cabo la fase II de esta propuesta es necesario tener información que permita valorar cada alternativa a la luz de los criterios definidos en la fase anterior. Para obtener dicha información se utilizan fuentes primarias y secundarias, las cuales permiten dar valores específicos para cada alternativa con relación a los criterios.

Los criterios cuantitativos tales como costo/ precio, experiencia (medida en años) o entregas a tiempo, pueden ser valorados a través de datos puntuales, obtenidos a través de proveedores, cotizaciones y/o experiencias de otros clientes.

Para el caso de los criterios cualitativos, se definen una serie de escalas de calificación que evalúan diferentes ítems. Esta información puede ser obtenida directamente por el proveedor o a través de información secundaria. Por ejemplo, para valorar el criterio "Calidad" se propone tener en cuenta las certificaciones que según los expertos son las más importantes al momento de seleccionar un proveedor. Estas son: ISO 28000: Sistemas de gestión de Seguridad para la Cadena de Suministro, la certificación BASC: Business Alliance for Secure Commerce y la ISO 9001Sistema de Gestión de Calidad. Además de las certificaciones, para este criterio también se propone tener en cuenta la antigüedad de la flota.

En la Tabla 4 se presenta la propuesta de valoración del criterio Calidad para las alternativas.

Se establece la matriz de decisión, a partir de la información obtenida en el paso anterior para $m$ alternativas y $n$ criterios. Cabe resaltar que los elementos de la matriz deben ser valores cuantitativos, para poder llevar a cabo la metodología.

\section{TABLA 4. VALORACIÓN DEL CRITERIO CALIDAD PARA LAS ALTERNATIVAS}

FUENTE: ELABORACIÓN PROPIA

\begin{tabular}{|c|c|c|c|c|c|}
\hline \multirow[b]{2}{*}{ Ítem } & \multicolumn{5}{|c|}{ ESCALA } \\
\hline & 1 & 2 & 3 & 4 & 5 \\
\hline Aspecto 1 & $\begin{array}{l}\text { No tiene } \\
\text { certificaciones }\end{array}$ & $\begin{array}{l}\text { No tiene } \\
\text { certificaciones, pero } \\
\text { está en proceso de } \\
\text { implementación y } \\
\text { certificación }\end{array}$ & $\begin{array}{l}\text { Tiene certificación } \\
\text { ISO 9001:2008 }\end{array}$ & $\begin{array}{l}\text { Tiene certificación } \\
\text { BASC }\end{array}$ & $\begin{array}{l}\text { Tiene certificación } \\
\text { ISO } 28000\end{array}$ \\
\hline Aspecto 2 & $\begin{array}{l}\text { Menos del } 20 \% \\
\text { de la flota tiene } \\
\text { menos de } 5 \text { años } \\
\text { de antigüedad }\end{array}$ & $\begin{array}{l}\text { Entre el } 20 \% \text { y } 40 \% \\
\text { de la flota tiene } \\
\text { menos de } 5 \text { años de } \\
\text { antigüedad }\end{array}$ & $\begin{array}{l}\text { Entre el } 40 \% \text { y } 60 \% \\
\text { de la flota tiene } \\
\text { menos de } 5 \text { años } \\
\text { de antigüedad }\end{array}$ & $\begin{array}{l}\text { Entre el } 60 \% \text { y } 80 \% \\
\text { de la flota tiene } \\
\text { menos de } 5 \text { años } \\
\text { de antigüedad }\end{array}$ & $\begin{array}{l}80 \% \text { de la flota } \\
\text { tiene menos } \\
\text { de } 5 \text { años de } \\
\text { antigüedad }\end{array}$ \\
\hline
\end{tabular}


Paso 7. Normalización de la matriz de decisión

El propósito de normalizar la matriz de decisión es convertir los criterios definidos en la fase I, en criterios adimensionales que puedan compararse entre sí. Para ellos se utilizará el método mostrado en la Ecuación 1

$$
r_{i j}=x_{i j} / \sqrt{\left(\sum x_{i j}^{2}\right)} \text { Para } i=1,2, \ldots, \mathrm{n}
$$

Paso 8. Construcción de la matriz de decisión normalizada ponderada

Cada elemento normalizado de la matriz se debe multiplicar por el vector de prioridad de los criterios definidos en la fase I. Esto dará como resultado la matriz de decisión normalizada ponderada.

Paso 9. Determinación de la solución ideal positiva y la solución ideal negativa

Una vez definidos los criterios de costo y beneficio, se procede a hallar la solución ideal positiva y negativa con base en las Ecuaciones 2 y 3.

$$
\begin{aligned}
\overline{\mathrm{A}}^{+}=\left\{\bar{v}_{1}^{+}, \ldots, \bar{v}_{n}^{+}\right\} & =\left\{\left(\max \bar{v}_{i j}, j \varepsilon J\right)\left(\min \bar{v}_{i j}, j \varepsilon J^{\prime}\right)\right\} \\
i= & 1,2, \ldots, \mathrm{m} \\
\overline{\mathrm{A}}^{-}=\left\{\bar{v}_{1}^{+}, \ldots, \bar{\nu}_{n}^{+}\right\} & =\left\{\left(\max \bar{v}_{i j}, j \varepsilon J\right)\left(\min \bar{v}_{i j}, j \varepsilon J^{\prime}\right)\right\} \\
i & =1,2, \ldots, \mathrm{m}
\end{aligned}
$$

Si el criterio es de costo, la solución ideal positiva será el valor mínimo de las alternativas con relación al criterio evaluado. Si el criterio es de beneficio, la solución ideal positiva será el valor máximo de las alternativas con relación al criterio evaluado.

Paso 10. Cálculo de las medidas de distancia

Después de definir la solución ideal positiva y la negativa, se debe calcular la distancia euclidiana entre estas soluciones y cada una de las alternativas.

Paso 11. Cálculo de la proximidad relativa a la solución ideal

Debe calcularse la proximidad relativa a la solución ideal aplicando la Ecuación 4.

$$
\bar{R} i=\frac{\bar{d}_{i}^{-}}{\bar{d}_{i}^{+}+\bar{d}_{i}^{-}}, i=1, \ldots, m(4)
$$

\section{Paso 12. Selección de alternativa}

Finalmente, se ordenan las mejores alternativas de acuerdo con el valor $\mathrm{R}_{\mathrm{i}}$ en orden descendente. Cuanto más próximo es este valor a 1 , implica una mayor prioridad de la alternativa i-ésima.

\section{Aplicación de la metodología propuesta para la selección de un proveedor 3PL en actividades de transporte}

La aplicación de la metodología se validó en una multinacional americana del sector papelero, presente en más de 30 países alrededor del mundo. Entre sus actividades principales se encuentran la fabricación de envases, productos industriales, soluciones de protección y servicios de exhibición y embalaje, con instalaciones en la ciudad de Cali y se enfocó para las actividades de trasporte tercerizado, siendo estas las más importantes dentro del grupo de las que se subcontratan en la organización.

Se realizaron cada uno de los pasos descritos anteriormente para la selección de proveedores logísticos. A continuación se detallan cada uno de ellos y el proceso correspondiente.

Paso 1. Selección del grupo de expertos y definición de los criterios

El grupo de expertos estuvo conformado por cinco personas relacionadas directamente con las actividades de transporte tercerizado, Estos fueron: El gerente de abastecimiento, el jefe nacional de compras, los dos jefes de despacho y el coordinador de exportaciones.

Los criterios a evaluar en el caso de estudio, fueron seleccionados por los expertos a través de la aplicación de un cuestionario. Dicho cuestionario comprendió los principales criterios utilizados para transporte en la selección de proveedores 3PL, según los autores revisados. Estos criterios fueron calificados por los expertos en una escala de 1 a 9 y de esta manera se conoció cuáles fueron los más relevantes en este campo. A continuación se describen cada uno de ellos: 
- Confiabilidad y reputación: Experiencia previa, honestidad, confidencialidad, estatus en el mercado, escala del proveedor y veracidad de la información.

- Calidad: Flota en buen estado, conductores entrenados, protocolos en manejo de carga, certificaciones.

- Costo/Precio: Costos logísticos, costos de operación, precios competitivos, ahorro en costos, plazos de pago.

- Entregas a tiempo: Ofrecer un producto o servicio que satisfaga las necesidades del cliente frente a un pliego de condiciones de tiempo de entrega. La entrega a tiempo se mide como el porcentaje de logro dentro de una ventana de tiempo que se establece entre la fecha solicitada por el cliente y la fecha comprometida por parte del proveedor.

- Servicio: Variedad de servicios, servicios de valor agregado, sistemas de información, amplia cobertura de pólizas, servicios de emergencia, servicio al cliente, cobertura geográfica.

- Experiencia: Años de trayectoria, conocimiento del proveedor de los servicios en la industria y puntualidad.
- Riesgo por falta de profesionalismo: El intercambio de información no autorizada con terceros, la incertidumbre en precios, poca flexibilidad en el servicio y la mala comunicación entre el usuario y el proveedor.

- Riesgos de calidad: Se asocia a documentación incorrecta o falsa y la falta de procedimientos, mercancía entregada en mal estado y en cantidades incorrectas.

Además de los criterios, los expertos definieron tres alternativas que soportaron el proceso de evaluación en el caso estudio. Tales alternativas fueron definidas como:

- $\mathrm{A}_{1}$ : Alternativa 1. Proveedor con más de 20 años en la industria de transporte terrestre de carga. Tiene su propia flota de vehículos. Cuenta con certificación ISO 28000.

- $\mathrm{A}_{2}$ : Alternativa 2. Proveedor con 27 años en la industria de transporte de carga terrestre. Cuenta con una flota moderna (alrededor del $60 \%$ tiene menos de 5 años). Certificado por BASC.

- $A_{3}$ : Alternativa 3. Proveedor con 50 años en la industria de transporte de carga terrestre. Más del $70 \%$ de su flota tiene menos de 5 años de antigüedad. Cuenta con certificación BASC.

\section{Figura 3. Representación Jerárquica para la selección de proveedores 3PL, según la propuesta metodológica}

Fuente: Elaboración propia

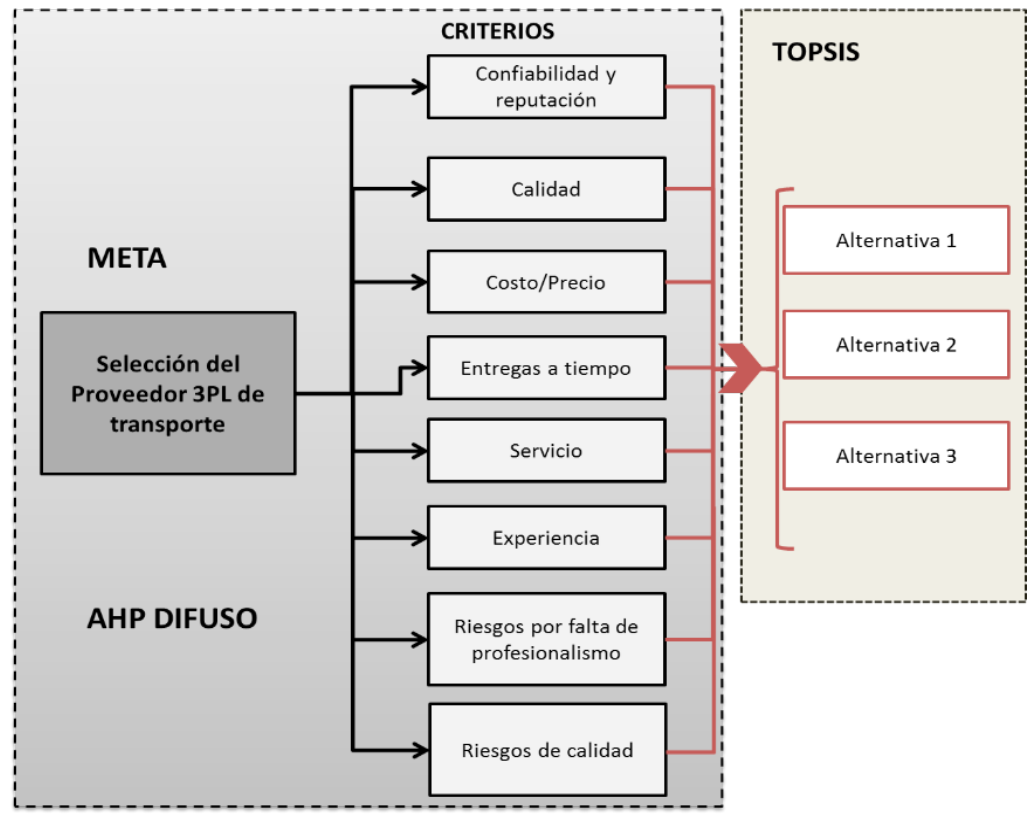




\begin{tabular}{|c|c|c|c|c|c|c|c|c|c|c|c|c|}
\hline & \multicolumn{3}{|c|}{$\begin{array}{l}\text { Confiabilidad y } \\
\text { reputación }\end{array}$} & \multicolumn{3}{|c|}{ Calidad } & \multicolumn{3}{|c|}{... } & \multicolumn{3}{|c|}{ Entregas a tiempo } \\
\hline Confiabilidad y reputación & 1 & 1 & 1 & $2 / 5$ & $1 / 2$ & $3 / 5$ & $\ldots$ & $\ldots$ & $\ldots$ & $3 / 4$ & $5 / 6$ & $12 / 5$ \\
\hline Calidad & $12 / 3$ & $21 / 6$ & $21 / 2$ & 1 & 1 & 1 & $\cdots$ & $\ldots$ & $\cdots$ & $11 / 7$ & $11 / 4$ & $22 / 7$ \\
\hline Riesgos de calidad & $3 / 5$ & $5 / 7$ & $7 / 8$ & $7 / 8$ & $11 / 5$ & $13 / 7$ & .. & $\ldots$ & $\ldots$ & $1 / 2$ & $2 / 3$ & $7 / 8$ \\
\hline $\begin{array}{l}\text { Riesgos por falta de } \\
\text { profesionalismo }\end{array}$ & $2 / 7$ & $1 / 3$ & $3 / 7$ & $1 / 4$ & $1 / 3$ & $2 / 5$ & $\cdots$ & $\cdots$ & $\cdots$ & $3 / 7$ & $1 / 2$ & $3 / 4$ \\
\hline Costo/ precio & $2 / 7$ & $3 / 7$ & $1 / 2$ & $1 / 2$ & $2 / 3$ & $7 / 8$ & $\ldots$ & $\ldots$ & $\ldots$ & $1 / 2$ & $4 / 7$ & 1 \\
\hline Servicio & $12 / 3$ & $21 / 2$ & $26 / 7$ & $1 / 3$ & $1 / 2$ & $3 / 5$ & ... & $\ldots$ & ... & 1 & $12 / 5$ & 2 \\
\hline Experiencia & $4 / 5$ & $11 / 8$ & $11 / 3$ & $1 / 5$ & $1 / 4$ & $1 / 3$ & $\ldots$ & $\ldots$ & $\ldots$ & $1 / 2$ & $4 / 7$ & 1 \\
\hline Entregas a tiempo & $5 / 7$ & $11 / 5$ & $11 / 3$ & $3 / 7$ & $4 / 5$ & $7 / 8$ & $\ldots$ & $\cdots$ & $\cdots$ & 1 & 1 & 1 \\
\hline
\end{tabular}

Una vez conocido el objetivo o meta, los criterios y las alternativas, se puede realizar la representación jerárquica propia para este caso estudio. Esta es presentada a través de la Figura 3.

\section{Paso 2. Construcción de las matrices di-} fusas de comparación con base en los juicios de los expertos

Las matrices difusas de comparación requieren ser construidas con base en los juicios de los expertos a través del uso de escalas lingüísticas. Para conocer sus opiniones, se aplicó un segundo cuestionario, en este se debía evaluar la preferencia de cada criterio respecto a otro. Esta comparación se desarrolló con base en la escala lingüística mostrada en la Tabla 5, en la que definieron si era "Igualmente preferido o importante", "Moderadamente preferido o importante", "Fuertemente preferido o importante", "Preferencia o importancia muy fuerte o demostrada" o "Extremadamente preferido o importante" un criterio sobre el otro.

Como existe más de un decisor, estos juicios deben ser consolidados a través de la media geométrica. Los elementos resultantes conforman la matriz difusa de comparación. En la Tabla 5 se presenta un fragmento de la matriz consolidada de comparaciones de los expertos.
Paso 3. Aplicación del método AHP difuso de Buckley

Según lo descrito en el paso 3 de la propuesta metodológica para selección, se desarrollaron los cálculos matemáticos descritos para definir los valores de " $r_{i}$ ", " $w_{i}$ " $y$ " $M_{i}$ " para obtener como resultado el vector de prioridad " $\mathrm{N}_{\mathrm{i}}$ " del peso de los criterios y el cual se presenta en la Tabla 6.

TABLA 6. VECTOR PRIORIDAD DE LOS PESOS DE LOS CRITERIOS PARA LA SELECCIÓN DE PROVEEDORES 3PL DE TRANSPORTE

FUENTE: ELABORACIÓN PROPIA

\begin{tabular}{l|c}
\multicolumn{1}{c|}{ Criterio } & $\mathbf{N}_{\mathbf{i}}$ \\
\hline Confiabilidad y reputación & $12,33 \%$ \\
\hline Calidad & $20,49 \%$ \\
\hline Riesgos de calidad & $11,93 \%$ \\
\hline Riesgos por falta de profesionalismo & $7,06 \%$ \\
\hline Costo/ precio & $6,83 \%$ \\
\hline Servicio & $17,23 \%$ \\
\hline Experiencia & $10,08 \%$ \\
\hline Entregas a tiempo & $14,05 \%$ \\
\hline
\end{tabular}


TABLA 6. MATRIZ DE DECISIÓN

FUENTE: ELABORACIÓN PROPIA

\begin{tabular}{|c|c|c|c|c|c|c|c|c|}
\hline CRITERIO & $\begin{array}{c}\text { Confiabilidad } \\
\text { y reputación }\end{array}$ & Calidad & $\begin{array}{c}\text { Costo/ } \\
\text { precio }\end{array}$ & $\begin{array}{c}\text { Entregas } \\
\text { a tiempo }\end{array}$ & Servicio & Experiencia & $\begin{array}{c}\text { Riesgos } \\
\text { por falta de } \\
\text { profesionalismo }\end{array}$ & $\begin{array}{c}\text { Riesgos } \\
\text { de } \\
\text { calidad }\end{array}$ \\
\hline $\begin{array}{c}\text { A1 } \\
\text { A2 }\end{array}$ & $100 \%$ & 4 & $\$ 1.300 .000$ & $98,30 \%$ & 3,33 & 20 años & 1 & 1,5 \\
\hline A3 & $75 \%$ & 3,5 & $\$ 1.250 .000$ & $95 \%$ & 3,67 & 27 años & 1,5 & 2 \\
\hline $\begin{array}{c}\text { Peso } \\
\text { de los } \\
\text { criterios }\end{array}$ & $12,33 \%$ & 4 & $\$ 1.400 .000$ & $90 \%$ & 3,33 & 50 años & 1,5 & 2 \\
\hline
\end{tabular}

TABLA 7. MATRIZ DE DECISIÓN NORMALIZADA PONDERADA

FUENTE: ELABORACIÓN PROPIA

\begin{tabular}{c|c|c|c|c|c|c|c|c} 
CRITERIO & $\begin{array}{c}\text { Confiabilidad } \\
\text { y reputación }\end{array}$ & Calidad & $\begin{array}{c}\text { Costo/ } \\
\text { precio }\end{array}$ & $\begin{array}{c}\text { Entregas } \\
\text { a tiempo }\end{array}$ & Servicio & Experiencia & $\begin{array}{c}\text { R. Falta de } \\
\text { profesionalismo }\end{array}$ & $\begin{array}{c}\text { R. } \\
\text { Calidad }\end{array}$ \\
\hline A1 & 0,079 & 0,123 & 0,039 & 0,084 & 0,096 & 0,033 & 0,030 & 0,056 \\
A2 & 0,059 & 0,108 & 0,037 & 0,082 & 0,106 & 0,045 & 0,045 & 0,075 \\
A3 & 0,075 & 0,123 & 0,042 & 0,077 & 0,096 & 0,084 & 0,045 & 0,075 \\
\hline
\end{tabular}

\section{Paso 4. Análisis de consistencia lógica}

Finalmente se halló la consistencia, dando un resultado de 0,08 . Dicho resultado afirma que, como la razón de consistencia es menor a 0,1, la matriz consolidada es consistente, y es correcto usar el vector prioridad obtenido inicialmente para este caso estudio.

Paso 5. Identificación y valloración de las alternativas

Las tres alternativas seleccionadas fueron valoradas con base en los criterios definidos en la fase anterior. Por lo tanto, fue necesario tener información previa relacionada con los criterios a evaluar.

Para ello se diseñó una escala de valoración para cada criterio seleccionado de la fase anterior la cual permitió evaluar objetivamente cada alternativa. Un ejemplo de esta valoración es el que se presentó en la Tabla 4.

\section{decisión \\ Paso 6. Establecimiento de la matriz de}

Una vez es obtenida y consolidada la información referente a cada alternativa, se procedió a construir la matriz de decisión con el fin de evaluar los tres provee- dores 3PL en función de los ocho criterios definidos. En la Tabla 6 se presenta la matriz de decisión.

\section{Paso 7. Normalización de la matriz de} decisión

Se aplicó la Ecuación 1 para normalizar la matriz descrita en el paso anterior. Como los elementos de esta matriz están expresados en diferentes unidades, la normalización permite obtener una matriz adimensional, la cual proporciona la comparación de los criterios entre sí.

Paso 8. Construcción de la matriz de decisión normalizada ponderada.

Posteriormente, cada elemento normalizado de la matriz se multiplicó por el vector de prioridad de los criterios obtenido en la Fase I, y así, se obtuvo la matriz de decisión normalizada ponderada la cual se presenta en la Tabla 7.

Paso 9. Determinación de la solución ideal positiva y la solución ideal negativa

Los criterios de costo y beneficio fueron definidos de tal manera que, para el caso de los criterios de costo, se consideraron los riesgos puesto que estos 
requieren ser minimizados. Con base en ello se procedió a hallar la solución ideal positiva y la solución ideal negativa aplicando las Ecuaciones 2 y 3 . En la Tabla 8 se presentan los resultados.

\section{Paso 10. Cálculo de las medidas de dis-} tancia

Se calculó la separación de cada alternativa respecto a la solución ideal positiva y a la separación ideal negativa.

\section{Paso 11. Cálculo de la proximidad relati-} va a la solución ideal

Posteriormente se realizó el cálculo de la proximidad relativa a la solución ideal. Los resultados se presentan en la Tabla 9.

\section{Paso 12. Selección de la alternativa}

Una vez obtenidos los pesos para cada una de las alternativas, estas se ordenaron de acuerdo al valor $\mathrm{R}_{\mathrm{i}}$ más cercano a 1. Los resultados obtenidos mostraron que la alternativa 3 se ajustó a la solución ideal positiva y por lo tanto satisface las necesidades de la compañía para este caso en particular.

\section{Conclusiones}

La metodología es un aporte valioso para las organizaciones y ello se ha logrado evidenciar en la aplicación del caso de estudio. Aquella les permitió, entre otras cosas, conocer el desempeño actual de algunos de sus proveedores, con el propósito de realizar actividades de retroalimentación y mejoramiento en los procesos de prestación del servicio de transporte tercerizado.

Debido al aumento de la globalización, las compañías se encuentran en un ambiente dinámico cada vez más competitivo expuesto a diversos factores de riesgo. La selección de aliados estratégicos es una labor continua de las empresas y por ello estas requieren tener procesos correctamente estructurados de selección y evaluación, que permitan mejorar la gestión de compras estratégica de la empresa reduciendo las probabilidades de ocurrencia de sucesos adversos. Con la aplicación del caso estudio se evidencia que la metodología propuesta puede ser incorporada como un proceso para diferentes compañías, puesto que este se presenta estructuradamente bajo fundamentos teóricos y es de fácil adaptabilidad.

\section{TABLA 8. CLASIFICACIÓN DE LOS CRITERIOS Y SOLUCIÓN IDEAL POSITIVA Y NEGATIVA}

\section{FUENTE: ELABORACIÓN PROPIA}

\begin{tabular}{c|c|c|c|c|c|c|c|c|}
\hline & $\begin{array}{c}\text { Confiabilidad } \\
\text { y reputación }\end{array}$ & Calidad & $\begin{array}{c}\text { Costo/ } \\
\text { precio }\end{array}$ & $\begin{array}{c}\text { Entregas } \\
\text { a tiempo }\end{array}$ & Servicio & Experiencia & $\begin{array}{c}\text { R. Falta de } \\
\text { profesionalismo }\end{array}$ & $\begin{array}{c}\text { R. } \\
\text { Calidad }\end{array}$ \\
\hline $\begin{array}{c}\text { Tipo de } \\
\text { criterio }\end{array}$ & Beneficio & Beneficio & Costo & Beneficio & Beneficio & Beneficio & Costo/Riesgo & $\begin{array}{c}\text { Costo/ } \\
\text { Riesgo }\end{array}$ \\
\hline $\begin{array}{c}\text { IDEAL } \\
\text { POSITIVA }\end{array}$ & 0,079 & 0,123 & 0,037 & 0,084 & 0,106 & 0,084 & 0,030 & 0,056 \\
\hline $\begin{array}{c}\text { IDEAL } \\
\text { NEGATIVA }\end{array}$ & 0,059 & 0,108 & 0,042 & 0,077 & 0,096 & 0,033 & 0,045 & 0,075 \\
\hline
\end{tabular}

TABLA 9. DISTANCIAS EUCLIDIANAS PARA LA SOLUCIÓN IDEAL POSITIVA Y NEGATIVA, CÁLCULO DEL Ri

FUENTE: ELABORACIÓN PROPIA

\begin{tabular}{c|c|c|c|} 
& D. Ideal positiva & D. Ideal Negativa & $\mathrm{R}_{\mathrm{i}}$ \\
\hline A1 & 0,051 & 0,035 & 0,404 \\
\hline A2 & 0,052 & 0,016 & 0,237 \\
\hline A3 & 0,027 & 0,055 & $\mathbf{0 , 6 6 8}$ \\
\hline
\end{tabular}


Los procesos de toma de decisiones que involucran diferentes partes de una organización, contienen un alto nivel de incertidumbre. El uso de una herramienta como el AHP difuso en estos procesos permite disminuir la subjetividad inmersa en los juicios emitidos por los expertos, ya que estos juicios pueden ser conocidos a través del uso de escalas lingüísticas que involucran números difusos triangulares.

\section{Referencias}

Aguezzoul, A., 2010. Multi-criteria decision making methods for Third-Party Logistics evaluation. Engineering Systems Management and Its Applications (ICESMA), 2010 Second International Conference on.

Aguezzoul, A., 2011. Overview on supplier selection of goods versus 3PL selection. 2011 th International Conference on Logistics, LOGISTIQUA'2011, pp.248-253.

Aguezzoul, A., 2014. Third-party logistics selection problem: A literature review on criteria and methods. Omega, [online] 49, pp.69-78. Available at: <http://www.sciencedirect.com/science/article/pii/ S0305048314000711>

Akman, G. and Baynal, K., 2014. Logistics Service Provider Selection through an Integrated Fuzzy Multicriteria Decision Making Approach. Journal of industrial Engineering, 2014.

Aqlan, F. and Lam, S.S., 2015. A fuzzy-based integrated framework for supply chain risk assessment. International Journal of Production Economics, [online] 161, pp.54-63. Available at: <http://linkinghub.elsevier. com/retrieve/pii/S0925527314003636>.

Arroyo, M., Gaytan, J. and Sierra, S., 2007. El proceso de toma de decisiones para la tercerización de funciones logísticas: prácticas mexicanas. ITESM, Toluca, (221), pp.1-28.

Ayhan, M.B., 2013. A Fuzzy AHP Approach for Supplier Selection Problem: A Case Study in a Gearmotor Company. Internation Journal of Managing Value and Supply Chains (IJMVSC), 4(3), pp.11-23.

Bansal, A. and Kumar, P., 2013. 3PL selection using hybrid model of AHP-PROMETHEE. International Journal of Services and Operations Management, 14(3), p.373.

Bansal, A., Kumar, P. and Issar, S., 2013. 3PL Selection : A Multi-criteria Decision Making Approach. Industrial Engineering and Engineering Management (IEEM), pp.981-985.

Bayazit, O. and Karpak, B., 2013. Selection of a third party logistics service provider for an aerospace company:
An analytical decision aiding approach. International Journal of Logistics Systems and Management, 15(4), pp.382-404.

Chan, F.T.S. and Kumar, N., 2007. Global supplier development considering risk factors using fuzzy extended AHP-based approach. Omega, 35, pp.417-431.

Chang, C.W., Wu, C.R. and Chen, H.C., 2008. Using expert technology to select unstable slicing machine to control wafer slicing quality via fuzzy AHP. Expert Systems with Applications, 34(3), pp.2210-2220.

Chang, D.-Y., 1996. Applications of the extent analysis method on fuzzy AHP. European Journal of Operational Research, 95(3), pp.649-655.

Chen, P.-S. and Wu, M.-T., 2013. A modified failure mode and effects analysis method for supplier selection problems in the supply chain risk environment: A case study. Computers \& Industrial Engineering, 66(4), pp.634-642.

Chunhao, L., Yonghe, S. and Yuanwei, D., 2008. Selection of 3PL service suppliers using a fuzzy analytic network process. Chinese Control and Decision Conference, 2008, CCDC 2008, pp.2174-2179.

Cooper, O., Tadikamalla, P. and Shang, J., 2012. Selection of a Third-Party Logistics Provider: Capturing the Interaction and In fl uence of Performance Metrics with the Analytical Network Process. 128(January), pp.115-128.

Dadeviren, M. and Yuksel, I., 2008. Developing a fuzzy analytic hierarchy process (AHP) model for behaviorbased safety management. Information Sciences, [online] 178(6), pp.1717-1733. Available at: <http:// dx.doi.org/10.1016/j.ins.2007.10.016>.

Daim, T.U., Udbye, A. and Balasubramanian, A., 2013. Use of analytic hierarchy process (AHP) for selection of 3PL providers. Journal of Manufacturing Technology Management, [online] 24(1), pp.28-51. Available at: <http://dx.doi.org/10.1108/17410381311287472>.

Datta, S., Samantra, C., Mahapatra, S.S., Mandal, G. and Majumdar, G., 2013. Appraisement and selection of third party logistics service providers in fuzzy environment. Benchmarking: An International Journal, [online] 20(4), pp.537-548. Available at: <http:// dx.doi.org/10.1108/BIJ-11-2011-0087>.

Demirel, T. and Kahraman, C., 2008. Fuzzy Analytic Hierarchy Process and its Application. Springer Optimization and Its Applications, 16, pp.53-83.

Govindan, K. and Chaudhuri, A., 2015. Interrelationships of risks faced by third party logistics service providers: A DEMATEL based approach. Transportation Research Part E: Logistics and Transportation Review. 
Govindan, K. and Murugesan, P., 2011. Selection of thirdparty reverse logistics provider using fuzzy extent analysis. Benchmarking: An International Journal, 18(1), pp.149-167.

Hassanzadeh, S. and Razmi, J., 2009. An integrated fuzzy model for supplier management: A case study of ISP selection and evaluation. Expert Systems with Applications, [online] 36(4), pp.8639-8648. Available at: <http://dx.doi.org/10.1016/j.eswa.2008.10.012>.

Ho, W., Dey, P.K. and Lockstrom, M., 2011. Strategic sourcing: a combined QFD and AHP approach in manufacturing. Supply Chain Management: An International Journal, 16(6), pp.446-461.

Ho, W., He, T., Lee, C.K.M. and Emrouznejad, A., 2012. Strategic logistics outsourcing: An integrated QFD and fuzzy AHP approach. Expert Systems with Applications, [online] 39(12), pp.10841-10850. Available at: <http:// dx.doi.org/10.1016/j.eswa.2012.03.009>.

Hosseininasab, A. and Ahmadi, A., 2015. Selecting a supplier portfolio with value, development, and risk consideration. European Journal of Operational Research, [online] 245(1), pp.146-156. Available at: <http:// dx.doi.org/10.1016/j.ejor.2015.02.041>.

Huo, B., Ye, Y. and Zhao, X., 2015. The impacts of trust and contracts on opportunism in the 3PL industry: The moderating role of demand uncertainty. International Journal of Production Economics, 170, pp.160-170.

Hwang, B.-N., Chen, T.-T. and Lin, J.T., 2016. 3PL selection criteria in integrated circuit manufacturing industry in Taiwan. Supply Chain Management: An International Journal, 21(1), pp.103-124.

Hwang, B.-N. and Shen, Y.-C., 2015. Decision Making for Third Party Logistics Supplier Selection in Semiconductor Manufacturing Industry: A Nonadditive Fuzzy Integral Approach. Mathematical Problems in Engineering, 2015(Mcdm), pp.1-12.

Jharkharia, S. and Shankar, R., 2007. Selection of logistics service provider: An analytic network process (ANP) approach. Omega, 35(3), pp.274-289.

Karsak, E.E. and Dursun, M., 2014. Expert Systems with Applications An integrated supplier selection methodology incorporating QFD and DEA with imprecise data. Expert Systems With Applications, [online] 41(16), pp.6995-7004. Available at: <http://dx.doi. org/10.1016/j.eswa.2014.06.020>.

Karthik B., Rakesh D. Raut, S.S. and Kamble, M.G.K. and S.J., 2015. Decision support system framework for performance based evaluation and ranking system of carry and forward agents. Strategic Outsourcing: An International Journal.
Kilincci, O. and Onal, S.A., 2011. Expert Systems with Applications Fuzzy AHP approach for supplier selection in a washing machine company. Expert Systems With Applications, [online] 38(8), pp.9656-9664. Available at: <http://dx.doi.org/10.1016/j.eswa.2011.01.159>.

Kull, T.J. and Talluri, S., 2008. A Supply Risk Reduction Model Using Integrated Multicriteria Decision Making. IEEE Transactions on Engineering Management, [online] 55(3), pp.409-419. Available at: <http://ieeexplore.ieee.org/lpdocs/epic03/wrapper. htm?arnumber $=4542978>$.

Kumar, P. and Singh, R.K., 2012. A fuzzy AHP and TOPSIS methodology to evaluate 3PL in a supply chain.Journal of Modelling in Management, 7(3), pp.287-303.

Lee, a. H.I., 2009. A fuzzy supplier selection model with the consideration of benefits, opportunities, costs and risks. Expert Systems with Applications, [online] 36(2), pp.2879-2893. Available at: <http://dx.doi. org/10.1016/j.eswa.2008.01.045>.

Lee, C.K.M., Ching Yeung, Y. and Hong, Z., 2012. An integrated framework for outsourcing risk management. Industrial Management \& Data Systems, [online] 112(4), pp.541-558. Available at: <http://www.emeraldinsight.com/doi/abs/10.1108/02635571211225477>.

Li, F., Li, L., Jin, C., Wang, R., Wang, H. and Yang, L., 2012. A 3PL supplier selection model based on fuzzy sets. Computers \& Operations Research, [online] 39(8), pp.18791884. Available at: <http://dx.doi.org/10.1016/j. cor.2011.06.022>.

Liu, H.T. and Wang, W.K., 2009. An integrated fuzzy approach for provider evaluation and selection in third-party logistics. Expert Systems with Applications, [online] 36(3), pp.4387-4398. Available at: <http:// dx.doi.org/10.1016/j.eswa.2008.05.030>.

Manotas Duque, D.F., Osorio Gómez, J.C. and Rivera, L., 2016. Operational Risk Assessment in Third Party Logistics.

Nekooie, M.A., Sheikhalishahi, M. and Hosnavi, R., 2015. Supplier selection considering strategic and operational risks: a combined qualitative and quantitative approach. Production Engineering, [online] 9(5-6), pp.665-673. Available at: <http://link.springer. com/10.1007/s11740-015-0643-6>.

Osorio, J.C., Manotas, D.F. and García, J.L., 2016. Multicriteria 3PL selection with risk consideration. Research in Computing Science, 109, pp.51-57.

Perçin, S., 2008. Fuzzy multi-criteria risk-benefit analysis of business process outsourcing (BPO). Information Management \& Computer Security, [online] 16(3), pp.213-234. Available at: <http://www.emeraldinsight.com/doi/abs/10.1108/09685220810893180>. 
Perçin, S., 2009. Evaluation of third-party logistics (3PL) providers by using a two-phase AHP and TOPSIS methodology. Benchmarking: An International Journal, 16(5), pp.588-604.

Pfohl, H., Gallus, P. and Thomas, D., 2011. Interpretive structural modeling of supply chain risks. International Journal of Physical Distribution \& Logistics Management, [online] 41(9), pp.839-859. Available at: <http://www.emeraldinsight.com/doi/ abs/10.1108/09600031111175816>.

Rattanawiboonsom, V., 2014. Effective Criteria for Selecting Third-Party logistics Providers : The Case of Thai Automotive Industry Rattanawiboonsom. 4(2), pp.196-205.

Saaty, R.W., 1987. The analytic hierarchy process-what it is and how it is used. Mathematical Modelling, 9(3-5), pp.161-176.

Sahu, N.K., Datta, S. and Mahapatra, S.S., 2015. Fuzzy based appraisement module for 3PL evaluation and selection.

Saleh Fahed Alkhatib, R.D. and T.T.N., 2015. Logistics Service Providers (LSPs) evaluation and selection: Literature review and framework development. Strategic Outsourcing: An International Journal.

Schoenherr, T., Rao Tummala, V.M. and Harrison, T.P., 2008. Assessing supply chain risks with the analytic hierarchy process: Providing decision support for the offshoring decision by a US manufacturing company. Journal of Purchasing and Supply Management, 14, pp.100-111.

Senthil, S., Srirangacharyulu, B. and Ramesh, A., 2012. A decision making methodology for the selection of reverse logistics operating channels. Procedia Engineering, 38, pp.418-428.

Shiang, K., Hung, H., Chuang, H. and Lin, W.H.C., 2011. An AHP-based approach to the selection of Third-Party Logistics. In: Decision Analysis Symposium. pp.1-11.

Soh, S., 2010. A decision model for evaluating third-party logistics providers using fuzzy analytic hierarchy process. African Journal of Business Management, [online] 4(3), pp.339-349. Available at: <http://academicjournals.org/AJBM/PDF/pdf2010/Mar/Soh.pdf>.

Tang, C.S., 2006. Perspectives in supply chain risk management. International Journal of Production Economics, 103, pp.451-488.

Tang, O. and Nurmaya Musa, S., 2011. Identifying risk issues and research advancements in supply chain risk management. International Journal of Production Economics, [online] 133(1), pp.25-34. Available at: <http:// dx.doi.org/10.1016/j.ijpe.2010.06.013>.
Urbano Guerrero, L.C., Muñoz Marín, L.S. and Osorio Gómez, J.C., 2015. Selección multicriterio de aliado estratégico para la operación de carga terrestre. Estudios Gerenciales, [online] 32(138), pp.35-43. Available at: <http://www.sciencedirect.com/science/article/pii/ S0123592315000601>.

Uygun, Ö., Kazamak, H. and Kahraman, Ü.A., 2014. An integrated DEMATEL and Fuzzy ANP techniques for evaluation and selection of outsourcing provider for a telecommunication company. Computers and Industrial Engineering, 86, pp.137-146.

Wang, Q. and Lv, H., 2015. Supplier Selection Group Decision Making in Logistics Service Value Cocreation Based on Intuitionistic Fuzzy Sets. Discrete Dynamics in Nature and Society, 2015, pp.1-10.

Wu, T., Blackhurst, J. and Chidambaram, V., 2006. A model for inbound supply risk analysis. Computers in Industry, 57, pp.350-365.

Zapata, J.A., 2011. Metodología para la implementación de tecnologías de la información y las comunicaciones TIC's para soportar una estrategia de cadena de suministro esbelta. TESIS DE MAESTRIA.
PARA CITAR ESTE ARTÍCULO / TO REFERENCE THIS ARTICLE / PARA CITAR ESTE ARTIGO /

Tabares-Urrea, N.; Ramírez-Flórez, G.; Osorio-Gómez, J.C. (2020). AHP difuso y TOPSIS para la selección de un proveedor 3PL considerando el riesgo operacional. Revista EIA, 17(33) enero-junio, Reia33007 pág. 1-17. Disponible en: https://doi.org/10.24050/reia.v17i33.1329 